\title{
Compressed Sensing Trilinear Model-based Angle Estimation for Bistatic MIMO radar
}

\author{
Xiaofei Zhang ${ }^{1,2}$, Jianfeng $\mathrm{Li}^{1}$, Ming Zhou ${ }^{1}$, De Ben ${ }^{1}$ \\ (1 Department of Electronic Engineering, Nanjing University of Aeronautics \& Astronautics, Nanjing, China, 210016) \\ (2.Nanjing Panda Electronics Group, Nanjing, China, 210002) \\ Email: fei_zxf@163.com
}

\begin{abstract}
In this paper, we address the transmit angle and receive angle estimation problem for a bistatic multiple-input multiple-output (MIMO) radar. This paper links MIMO radar angle estimation problem to the compressed sensing trilinear model. Exploiting this link, it derives a compressed sensing trilinear model-based angle estimation algorithm, which can obtain automatically paired two-dimensional angle estimation. The proposed algorithm requires no spectral peak searching or pair matching, and it has better angle estimation performance than conventional algorithms including estimation of signal parameters via rotational invariance techniques (ESPRIT) algorithm. Simulation results illustrate performance of the algorithm.
\end{abstract}

Index Terms-Array signal processing, angle estimation, multiple-input multiple-output (MIMO) radar, trilinear model, Compressed Sensing.

\section{INTRODUCTION}

Since multiple-input multiple-output (MIMO) radars use multiple antennas to simultaneously transmit diverse waveforms and utilize multiple antennas to receive the reflected signals, they have many potential advantages over conventional phased-array radar [1-5]. Transmit angle and receive angle estimation algorithms for MIMO radar have been investigated in Refs.[6-12]. In Ref. [6], estimation of signal parameters via rotational invariance techniques (ESPRIT) algorithms exploited the invariance property of both the transmit array and the receive array for direction estimation in MIMO radar systems. Ref. [7] presented another ESPRIT algorithm without pairing, whose complexity was lower than Ref. [6], but they both have almost the same angle estimation performance. Parallel factor (PARAFAC) algorithm has been used for detection and localization of multiple targets in a MIMO radar system $[8,9]$, and adaptive PARAFAC algorithm was proposed in [10] to support on-line applications. In this paper, we propose a compressed sensing trilinear model-based angle estimation algorithm, whose angle estimation performance is better than ESPRIT algorithm.

It is well known that a large number of signal processing methods are based on the theory of matrix or the bilinear model [12]. In general, matrix decomposition is not unique, since inserting a product of an arbitrary invertible matrix and its inverse in between two matrix factors preserves their product. Matrix decomposition can be unique only if one imposes additional structural properties including orthogonality, Vandermonde, Toeplitz, constant modulus and finite-alphabet constraints. Compared with the case of matrices, trilinear model or trilinear decomposition has a distinctive and attractive feature: it is often unique [12]. The uniqueness of trilinear decomposition is of great practical significance, which is important in many applications such as psychometrics, chemistry and biology. In the signal processing field, trilinear decomposition can be thought of as a generalization for ESPRIT and joint approximate diagonalization [13-15].

Compressive sensing [16-17] has attracted a lot of attention in the last decade, and it has been applied to a variety of problems, including image reconstruction and restoration, wavelet denoising, feature selection in machine learning, radar imaging and penalized regression [18]. The super-resolution property and ability of resolving coherent sources can be achieved when apply it in the source location [19]. Lots of the location methods using compressive sensing just use one snapshot and are very sensitive to the noise, for multiple snapshots, $\ell_{1}$-SVD method [18] employs $\ell_{1}$ norm to enforce sparsity and singular value decomposition to reduce complexity and sensitivity to noise, and sparse recovery for weighted subspace fitting (SRWSF) [19] improved the $\ell_{1}$-SVD method via the weight to the subspace. However, both the method in [18] and [19] have a common problem, which is the choice of the regularization parameter, and so a prior knowledge of the noise may be known.

This paper links the problem of MIMO radar angle estimation to the compressed sensing trilinear model. Exploiting this link, it derives the compressed sensing trilinear model-based angle estimation algorithm for MIMO radar, which has better angle estimation performance than ESPRIT algorithm. The proposed algorithm can obtain automatically paired two-dimensional angle estimation. Simulation results illustrate performance of the proposed algorithm.

Notation: $(.)^{*},(.)^{T},(.)^{H},(.)^{-1}$ and $(.)^{+}$denote complex conjugation, transpose, conjugate-transpose, inverse, pseudo-inverse operations, respectively. \|\|$_{F}$ presents Forbenius norm; $\operatorname{diag}(\mathbf{v})$ stands for diagonal matrix whose diagonal is a vector $\mathbf{v} ; \mathbf{I}_{K}$ is a $K \times K$ identity matrix; $\otimes$ and $\circ$ are the Kronecker product and Khatri-Rao product, respectively; $\min ($.) is to get minimum elements of an array; angle(b) is to get the phase angles for each element of complex array b. $D_{n}(\mathbf{A})$ stands for a diagonal matrix constructed out of the $n$th row of $\mathbf{A}$. 


\section{II.DATA MODEL}

We consider a bistatic MIMO radar system with array antennas containing an $M$-element-array transmitter and an $N$-element-array receiver, both of which are uniform linear arrays (ULAs) with half-wavelength spacing. We assume that there are $K$ uncorrelated targets, and the output of the matched filters at the receiver can be expressed as [6]

$$
\mathbf{x}(t)=\left[\mathbf{a}_{r}\left(\phi_{1}\right) \otimes \mathbf{a}_{t}\left(\theta_{1}\right), \cdots, \mathbf{a}_{r}\left(\phi_{K}\right) \otimes \mathbf{a}_{t}\left(\theta_{K}\right)\right] \mathbf{b}(t)
$$

where $\theta_{k}, \phi_{k}$ are the transmit angle and receive angle of the $k$ th targetl, respectively; $\mathbf{b}(t)=\left[b_{1}(t), b_{2}(t), \cdots, b_{K}(t)\right]^{T}$, and $b_{k}(t)=\beta_{k} e^{j 2 \pi f_{k} t}$ with $f_{k}$ being Doppler frequency and $\beta_{k}$ the amplitude. $\mathbf{a}_{r}\left(\phi_{k}\right) \otimes \mathbf{a}_{t}\left(\theta_{k}\right)$ is the Kronecker product of the receive and the transmit steering vectors for the $k$ th target. We assume $\mathbf{a}_{r}\left(\phi_{k}\right)$ and $\mathbf{a}_{t}\left(\theta_{k}\right)$ are constant for $L$ samples, and define $\mathbf{X}=[\mathbf{x}(1), \mathbf{x}(2), \cdots, \mathbf{x}(L)]$, which can be expressed as

$$
\mathbf{X}=\left[\mathbf{A}_{R} \circ \mathbf{A}_{T}\right] \mathbf{B}^{T}=\left[\begin{array}{c}
\mathbf{X}_{1} \\
\mathbf{X}_{2} \\
\vdots \\
\mathbf{X}_{N}
\end{array}\right]=\left[\begin{array}{c}
\mathbf{A}_{T} D_{1}\left(\mathbf{A}_{R}\right) \\
\mathbf{A}_{T} D_{2}\left(\mathbf{A}_{R}\right) \\
\vdots \\
\mathbf{A}_{T} D_{N}\left(\mathbf{A}_{R}\right)
\end{array}\right] \mathbf{B}^{T}
$$

where $\quad \mathbf{A}_{T}=\left[\mathbf{a}_{t}\left(\theta_{1}\right), \mathbf{a}_{t}\left(\theta_{2}\right), \ldots, \mathbf{a}_{t}\left(\theta_{K}\right)\right] \in \mathbb{C}^{M \times K} \quad$ and $\mathbf{A}_{R}=\left[\mathbf{a}_{r}\left(\phi_{1}\right), \mathbf{a}_{r}\left(\phi_{2}\right), \cdots, \mathbf{a}_{r}\left(\phi_{K}\right)\right] \in \mathbb{C}^{N \times K}$ are the transmit direction matrix and the receive direction matrix, respectively. $\quad \mathbf{A}_{R} \circ \mathbf{A}_{T} \quad$ is Khatri-Rao product. $\mathbf{B}=[\mathbf{b}(1), \mathbf{b}(2), \cdots, \mathbf{b}(L)]^{T} \in \mathbb{C}^{L \times K}$. Thus, $\mathbf{X}_{n}$ in Eq.(2) is denoted as

$$
\mathbf{X}_{n}=\mathbf{A}_{T} D_{n}\left(\mathbf{A}_{R}\right) \mathbf{B}^{T}, n=1,2, \ldots, N
$$

In the presence of noise, the signal model becomes $\tilde{\mathbf{X}}_{n}=\mathbf{A}_{T} D_{n}\left(\mathbf{A}_{R}\right) \mathbf{B}^{T}+\mathbf{V}_{n}, n=1,2, \ldots, N$, where $\mathbf{V}_{n}$ is the received noise of the $n$th slice. Eq.(3) is also denoted with the trilinear model [12]

$$
\begin{gathered}
x_{m, n, l}=\sum_{k=1}^{K} \mathbf{A}_{T}(m, k) \mathbf{A}_{R}(n, k) \mathbf{B}(l, k), \\
m=1, \cdots, M, n=1, \cdots, N, l=1, \cdots, L
\end{gathered}
$$

where $\mathbf{A}_{T}(m, k)$ stands for the $(m, k)$ element of the matrix $\mathbf{A}_{T}$, and similarly for the others. $\mathbf{X}_{n}=\mathbf{A}_{T} D_{n}\left(\mathbf{A}_{R}\right) \mathbf{B}^{T}(n=1, \ldots, N)$ can be interpreted as slicing the three dimension data in a series of slices along the spatial direction. The symmetry of the trilinear model in (4) allows two more matrix system rearrangements, in which we have $\mathbf{Y}_{m}=\mathbf{B} D_{m}\left(\mathbf{A}_{T}\right) \mathbf{A}_{R}{ }^{T}, m=1, \ldots, M$, and $\mathbf{Z}_{l}=\mathbf{A}_{R} D_{l}(\mathbf{B}) \mathbf{A}_{T}{ }^{T}, l=1, \ldots, L$. According to the symmetry of the trilinear model, we form the matrices of $\mathbf{Y}$ and $\mathbf{Z}$ as follows

$$
\mathbf{Y}=\left[\begin{array}{c}
\mathbf{Y}_{1} \\
\mathbf{Y}_{2} \\
\vdots \\
\mathbf{Y}_{M}
\end{array}\right]=\left[\mathbf{A}_{T} \circ \mathbf{B}\right] \mathbf{A}_{R}{ }^{T}
$$

$$
\mathbf{Z}=\left[\begin{array}{c}
\mathbf{Z}_{1} \\
\mathbf{Z}_{2} \\
\vdots \\
\mathbf{Z}_{L}
\end{array}\right]=\left[\mathbf{B} \circ \mathbf{A}_{R}\right] \mathbf{A}_{T}{ }^{T}
$$

We link the problem of MIMO radar angle estimation with compressed sensing trilinear model and derive compressed sensing trilinear model-based angle estimation algorithm, which is proposed and discussed in detail in the following section.

\section{III.COMPRESSED SENSING TRILINEAR MODEL}

\section{A.Compression}

As a first step, we compresses the three-way array $\overline{\mathbf{X}} \in \mathbb{C}^{M \times N \times L}$ into a (usually much) smaller three-way array $\overline{\mathbf{X}}^{\prime} \in \mathbb{C}^{M^{\prime} \times N^{\prime} \times L^{\prime}}$, where $M^{\prime}<M, \quad N^{\prime}<N$ and $L^{\prime}<L$.

We define the compressed matrices as $\mathbf{U} \in \mathbb{C}^{M \times M^{\prime}}$, $\mathbf{V} \in \mathbb{C}^{N \times N^{\prime}}$ and $\mathbf{W} \in \mathbb{C}^{L \times L^{\prime}}$, which can be obtained after the tucker3 model [20].

And then the compressed three way array can be expressed as

$$
\mathbf{X}^{\prime}=\left[\mathbf{A}_{R}^{\prime} \circ \mathbf{A}_{T}^{\prime}\right] \mathbf{B}^{\prime T}
$$

where $\quad \mathbf{A}_{T}^{\prime}=\mathbf{U}^{H} \mathbf{A}_{T}, \quad \mathbf{A}_{R}^{\prime}=\mathbf{V}^{H} \mathbf{A}_{R} \quad$ and $\quad \mathbf{B}^{\prime}=\mathbf{W}^{H} \mathbf{B}$. With respect to (5) and (6), we form the matrices of $\mathbf{Y}^{\prime}$ and $\mathbf{Z}^{\prime}$ according to the compressed data

$$
\begin{aligned}
& \mathbf{Y}^{\prime}=\left[\mathbf{A}_{T}^{\prime} \circ \mathbf{B}^{\prime}\right] \mathbf{A}_{R}^{\prime T} \\
& \mathbf{Z}^{\prime}=\left[\mathbf{B}^{\prime} \circ \mathbf{A}_{R}^{\prime}\right] \mathbf{A}_{T}^{\prime T}
\end{aligned}
$$

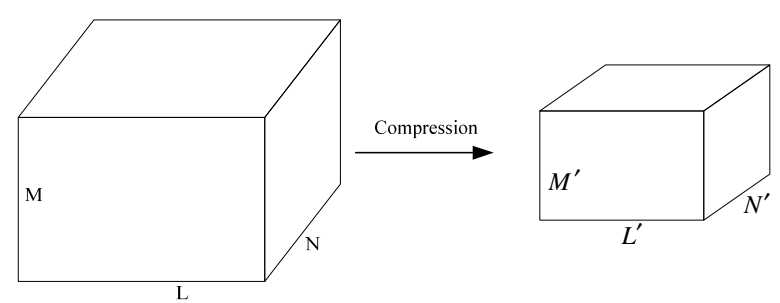

Fig. 1 the compression of trilinear model

\section{B.Trilinear Decomposition}

Trilinear alternating least square (TALS) algorithm is a common data detection method for the trilinear model [12]. The principle of TALS can be adopted to fit low rank trilinear models. We concisely show the basic idea behind TALS for three major steps: 1) Update one matrix each time using LS, which is conditioned on previously obtained estimates for the remaining matrices; 2) Proceed to update the other matrices; 3) Repeat until convergence of the LS cost function. TALS algorithm is discussed as follows.

According to (7), least squares (LS) fitting is

$$
\min _{\mathbf{A}_{T}^{\prime}, \mathbf{A}_{R}^{\prime}, \mathbf{B}^{\prime}}\left\|\tilde{\mathbf{X}}^{\prime}-\left[\mathbf{A}_{R}^{\prime} \circ \mathbf{A}_{T}^{\prime}\right] \mathbf{B}^{\prime T}\right\|_{F}
$$

where $\tilde{\mathbf{X}}^{\prime}$ is the noisy compressed signal. LS update for the matrix $\mathbf{B}^{\prime}$ is 


$$
\hat{\mathbf{B}}^{\prime T}=\left[\hat{\mathbf{A}}_{R}^{\prime} \circ \hat{\mathbf{A}}_{T}^{\prime}\right]^{+} \tilde{\mathbf{X}}^{\prime}
$$

where $\hat{\mathbf{A}}_{T}^{\prime}$ and $\hat{\mathbf{A}}_{R}^{\prime}$ are previously obtained estimates of $\mathbf{A}_{T}^{\prime}$ and $\mathbf{A}_{R}^{\prime}$, respectively. According to Eq.(8), LS fitting is

$$
\min _{\mathbf{A}_{T}^{\prime}, \mathbf{A}_{R}^{\prime}, \mathbf{B}^{\prime}}\left\|\tilde{\mathbf{Y}}^{\prime}-\left[\mathbf{A}_{T}^{\prime} \circ \mathbf{B}^{\prime}\right] \mathbf{A}_{R}^{\prime T}\right\|_{F}
$$

where $\tilde{\mathbf{Y}}^{\prime}$ is the noisy compressed signal. LS update for $\mathbf{A}_{R}^{\prime}$ is

$$
\hat{\mathbf{A}}_{R}^{\prime T}=\left[\hat{\mathbf{A}}_{T}^{\prime} \circ \hat{\mathbf{B}}^{\prime}\right]^{+} \tilde{\mathbf{Y}}^{\prime}
$$

where $\hat{\mathbf{A}}_{T}^{\prime}$ and $\hat{\mathbf{B}}^{\prime}$ stand for the previously obtained estimates of $\mathbf{A}_{T}^{\prime}$ and $\mathbf{B}^{\prime}$. Similarly, according to Eq.(9), LS fitting is

$$
\min _{\mathbf{A}_{T}^{\prime}, \mathbf{A}_{R}^{\prime}, \mathbf{B}^{\prime}}\left\|\tilde{\mathbf{Z}}^{\prime}-\left[\mathbf{B}^{\prime} \circ \mathbf{A}_{R}^{\prime}\right] \mathbf{A}_{T}^{\prime T}\right\|_{F}
$$

where $\tilde{\mathbf{Z}}^{\prime}$ is the noisy compressed signal. LS update for $\mathbf{A}_{T}^{\prime}$ is

$$
\hat{\mathbf{A}}_{T}^{\prime T}=\left[\hat{\mathbf{B}}^{\prime} \circ \hat{\mathbf{A}}_{R}^{\prime}\right]^{+} \tilde{\mathbf{Z}}^{\prime}
$$

where $\hat{\mathbf{A}}_{R}^{\prime}$ and $\hat{\mathbf{B}}^{\prime}$ stand for the previously obtained estimates of $\mathbf{A}_{R}^{\prime}$ and $\mathbf{B}^{\prime}$, respectively. According to (11), (13) and (15), the matrices $\mathbf{B}^{\prime}, \mathbf{A}_{R}^{\prime}$ and $\mathbf{A}_{T}^{\prime}$ are updated with least squares, respectively. The matrix update is repeated until convergence.

\section{C.Angle estimation with Sparsity}

After the trilinear decomposition, the estimation of $\mathbf{A}_{T}^{\prime}$, $\mathbf{A}_{R}^{\prime}$ and $\mathbf{B}^{\prime}$ can be obtained. Use $\mathbf{a}_{T k}^{\prime}$ and $\mathbf{a}_{R k}^{\prime}$ to denote the k-th column of $\mathbf{A}_{T}^{\prime}, \mathbf{A}_{R}^{\prime}$, respectively. And

$$
\mathbf{a}_{T k}^{\prime}=\mathbf{U}^{H} \mathbf{a}_{T k} \alpha_{T k}, \mathbf{a}_{R k}^{\prime}=\mathbf{V}^{H} \mathbf{a}_{R k} \alpha_{R k}
$$

where $\mathbf{a}_{T k}$ and $\mathbf{a}_{R k}$ are the $k$-th column of $\mathbf{A}_{T}, \mathbf{A}_{R}$, respectively. $\alpha_{T k}$ and $\alpha_{R k}$ denote the scaling coefficients.

Let $\tilde{\theta}_{1}, \tilde{\theta}_{2}, \cdots, \tilde{\theta}_{P}$ and $\tilde{\phi}_{1}, \tilde{\phi}_{2}, \cdots, \tilde{\phi}_{Q}$ be a sampling grid of all transmit angle and receive angle, respectively. $P>>K$ and $Q>>K$. We construct a matrix composed of steering vectors corresponding to each potential target location as its columns: $\quad \boldsymbol{\Theta}_{T}=\left[\mathbf{a}_{t}\left(\tilde{\theta}_{1}\right), \mathbf{a}_{t}\left(\tilde{\theta}_{2}\right), \cdots, \mathbf{a}_{t}\left(\tilde{\theta}_{P}\right)\right] \quad$ and $\boldsymbol{\Theta}_{R}=\left[\mathbf{a}_{r}\left(\tilde{\phi}_{1}\right), \mathbf{a}_{r}\left(\tilde{\phi}_{2}\right), \cdots, \quad \mathbf{a}_{r}\left(\tilde{\phi}_{Q}\right)\right]$. If $\tilde{\theta}_{p}=\theta_{k}$, then $\mathbf{a}_{T k}=\boldsymbol{\Theta}_{T} \mathbf{e}_{p}$, with $\mathbf{e}_{p}$ being $P \times 1$ sparse vector, the p-th element is 1 and others are zeros. Similarly for the receive angles.

Then the transmit angle and receive angle can be obtained via $l_{0}$-norm constraint,

$$
\begin{aligned}
& \hat{\theta}_{k}=\min \left\|\mathbf{a}_{T k}^{\prime}-\mathbf{U}^{H} \mathbf{a}_{t}\left(\tilde{\theta}_{p}\right)\left(\mathbf{U}^{H} \mathbf{a}_{t}\left(\tilde{\theta}_{p}\right)\right)^{+} \mathbf{a}_{T k}^{\prime}\right\|_{F}, p=1, \ldots, P \\
& \hat{\phi}_{k}=\min \left\|\mathbf{a}_{R k}^{\prime}-\mathbf{V}^{H} \mathbf{a}_{r}\left(\hat{\phi}_{q}\right)\left(\mathbf{V}^{H} \mathbf{a}_{r}\left(\hat{\phi}_{q}\right)\right)^{+} \mathbf{a}_{R k}^{\prime}\right\|_{F}, q=1, \ldots, Q
\end{aligned}
$$

As the columns of the estimated matrix are automatically paired, then the angle estimations can be automatically paired.

\section{D.Complexity analysis and $C R B$}

The proposed algorithm has lower computational complexity than trilinear decomposition-based method. The proposed algorithm reqiures $O\left(K^{3}+M^{\prime} N L^{\prime} K^{\prime}\right)$, while the trilinear decomposition algorithm needs $O\left(K^{3}+M N L K\right)$.

According to Ref.[21], we can derive the CRB in bistatic MIMO radar

$$
C R B=\frac{\sigma^{2}}{2 J}\left\{\operatorname{Re}\left[\mathbf{D}^{H} \boldsymbol{\Pi}_{\mathbf{A}}^{\perp} \mathbf{D} \odot \hat{\mathbf{P}}_{s}^{T}\right]\right\}^{-1}
$$

where $\mathbf{A}=\left[\mathbf{a}_{r}\left(\theta_{1}\right) \otimes \mathbf{a}_{t}\left(\theta_{1}\right)_{\gamma^{\prime}} \cdots, \mathbf{a}_{r}\left(\theta_{K}\right) \otimes \mathbf{a}_{t}\left(\theta_{K}\right)\right], \mathbf{a}_{k}$ the $k$ th column of $\mathbf{A}$, and; $\hat{\mathbf{P}}_{s}=\frac{1}{L} \sum \mathbf{b}(t) \mathbf{b}^{H}(t) ; \sigma^{2}$ is the noise power. $\boldsymbol{\Pi}_{\mathbf{A}}^{\perp}=\mathbf{I}_{M N}-\mathbf{A}\left(\mathbf{A}^{H} \mathbf{A}\right)^{L}{ }_{t=\mathbf{A}^{H}}^{H}$,

$$
\mathbf{D}=\left[\frac{\partial \mathbf{a}_{1}}{\partial \theta_{1}}, \frac{\partial \mathbf{a}_{2}}{\partial \theta_{2}}, \ldots, \frac{\partial \mathbf{a}_{K}}{\partial \theta_{K}}, \frac{\partial \mathbf{a}_{1}}{\partial \phi_{1}}, \frac{\partial \mathbf{a}_{2}}{\partial \phi_{2}}, \ldots, \frac{\partial \mathbf{a}_{K}}{\partial \phi_{K}}\right]
$$

\section{IV.SIMULATION RESULTS}

Define root mean squared error (RMSE) of the angles as $\frac{1}{K} \sum_{k=1}^{K} \sqrt{\frac{1}{500} \sum_{l=1}^{500}\left[\left(\hat{\theta}_{k, l}-\theta_{k}\right)^{2}+\left(\hat{\phi}_{k, l}-\phi_{k}\right)^{2}\right]}$, where $\hat{\theta}_{k, l} / \hat{\phi}_{k, l}$ is the estimate of DOD / DOA $\left(\theta_{k} / \phi_{k}\right)$ of the l-th Monte Carlo trial. In the following simulations, we normally adopt the bistatic MIMO array system with $M=8$ and $N=6$, and assume that there are three non-coherent targets with their angles correspondingly distributed as $\left(\theta_{1}, \phi_{1}\right)=\left(10^{\circ}, 20^{\circ}\right)$, $\left(\theta_{2}, \phi_{2}\right)=\left(-8^{\circ}, 30^{\circ}\right)$, and $\left(\theta_{3}, \phi_{3}\right)=\left(0^{\circ}, 45^{\circ}\right)$, respectively. The RCSs and Doppler frequencies are $\left(\beta_{1}, \beta_{2}, \beta_{3}\right)=[0.8,0.5,0.6] \quad$ and $\quad\left(f_{1}, f_{2}, f_{3}\right)=$ $[100 \mathrm{~Hz}, 2550 \mathrm{~Hz}, 5000 \mathrm{~Hz}]$, respectively.

Figs. 2 depict angle estimation result of the proposed algorithm for all three targets with SNR(Signal-to-Noise Ratio $)=0 \mathrm{~dB}$, respectively. It is shown that the DODs and DOAs can be clearly observed.

Fig. 3 presents the comparison between the proposed algorithm, the ESPRIT algorithm, the conventional trilinear decomposition-based method and CRB. From Fig. 4, we can find that the angle estimation performance of the proposed algorithm is better than that of ESPRIT algorithm, and has almost the same performance as that of the conventional trilinear decomposition-based method.

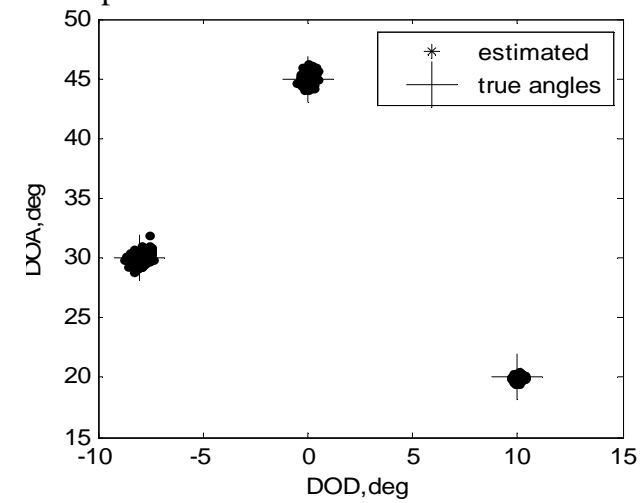

Fig.2 Angle estimation result with $\mathrm{SNR}=0 \mathrm{~dB}$ 


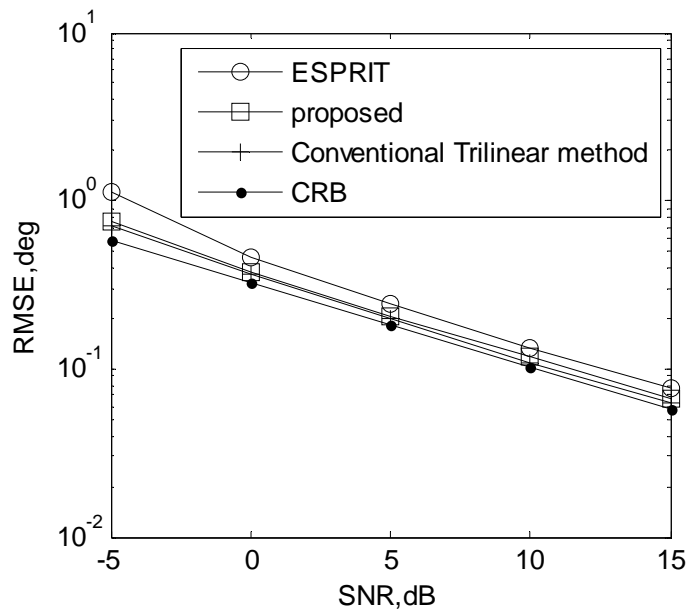

Fig.3 Angle estimation performance comparison

Fig.4 shows the performance comparison versus number of samples, in which SNR=20dB are used. It is indicated in Fig. 5 that the angle estimation performance of the proposed algorithm is better than ESPRIT and is little worse than conventional trilinear decomposition-based method and the angle estimation performances of the algorithms are improved when $L$ increases.

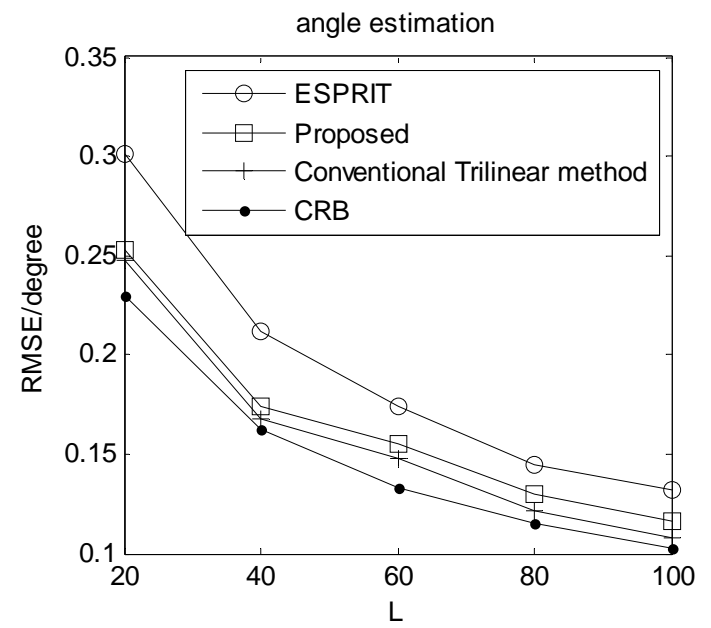

Fig.4 Angle estimation comparison for three targets versus $L$

\section{CONCLUSION}

In this paper, we link MIMO radar angle estimation problem to the compressed sensing trilinear model. Exploiting this link, it derives a compressed sensing trilinear model-based angle estimation algorithm, which can obtain automatically paired two-dimensional angle estimation. The proposed algorithm requires no spectral peak searching or pair matching, and it has better angle estimation performance than ESPRIT algorithm and has almost the same performance as that of conventional trilinear decomposition-based method.

\section{ACKNOWLEDGEMENTS}

This work is supported by China NSF Grants (61271327,61071164), Jiangsu Planned Projects for Postdoctoral Research Funds (1201039C), China Postdoctoral Science Foundation (2012M521099), Open project of key laboratory of underwater acoustic communication and marine information technology (Xiamen University), Hubei Key Laboratory of Intelligent Wire1ess Communications (IWC2012002), Open project of Key Laboratory of Nondestructive Testing (Nanchang Hangkong University), Open project of Key Laboratory of modern acoustic of Ministry of Education (Nanjing University), the Aeronautical Science Foundation of China (20120152001), by Qing Lan Project and the Fundamental Research Funds for the Central Universities (NZ2012010, kfjj120115, kfjj20110215).

\section{REFERENCES}

[1] E. Fishler, A. Haimovich, R. S. Blum, L. J. Cimini, D. Chizhik, and R. A. Valenzuela, "MIMO radar: an idea whose time has come," in Proc. IEEE Radar Conf., Apr. 2004, pp. 71-78.

[2] J. Li and P. Stoica, "MIMO radar-diversity means superiority," in Proc.14th Adaptive Sensor Array Process. Workshop (ASAP '06), Lincoln Lab, Mass, USA, Dec. 2006.

[3] E. Fishler, A. Haimovich, R. S. Blum, L. J. Cimini Jr., D. Chizhik, and R. A. Valenzuela, "Spatial diversity in radars models and detection performance,” IEEE Trans. Signal Process., vol. 54, no. 3, pp. 823-838, 2006.

[4] D. R. Fuhrmann and G. S. Antonio, "Transmit beamforming for MIMO radar systems using partial signal correlation," in Proc.38th Asilomar Conf. on Signals, Systems and Computers, Nov. 2004, vol. 1, pp. 295-299.

[5] L. Xu, J. Li, and P. Stoica, “Adaptive techniques for MIMO radar,” in Proc 4th Workshop on Sensor Array and Multichannel Signal Process., pp. 258-262, Jul. 2006.

[6] C. Duofang, C. Baixiao, and Q. Guodong, "Angle estimation using ESPRIT in MIMO radar,” Electron. Lett., vol. 44, no. 12, pp. 770-771, 2008.

[7] C. Jinli, G. Hong and S. Weimin, “Angle estimation using ESPRIT without pairing in MIMO radar,” Electron. Lett., vol. 44, no. 24, pp. 1422-1423, 2008

[8] D. Nion, N. D. Sidiropoulos, "A PARAFAC-based technique for detection and localization of multiple targets in a MIMO radar system," 2009 IEEE International Conference on Acoustics, Speech and Signal Processing, pp.2077-2080.

[9] X. Zhang, Z. Xu, L. Xu and D. Xu, "Trilinear decomposition-based transmit angle and receive angle estimation for multiple-input multiple-output radar", IET Radar, Sonar and Navigation, vol .5, no. 6, pp.626-631, 2011.

[10] D. Nion, N.D. Sidiropoulos, "Adaptive algorithms to track the PARAFAC decomposition of a third-order tensor," IEEE Transactions on Signal Processing, vol. 57, no. 6, pp.2299-2310, Jun. 2009

[11] M. Jin, G Liao and J. Li, "Joint DOD and DOA estimation for bistatic MIMO radar," Signal Processing, vol. 89, pp. 244-251, 2009.

[12] J. B. Kruskal, "Three-way arrays: rank and uniqueness of trilinear decompositions, with application to arithmetic complexity and statistics," Linear Algebra Applicat., vol. 18, pp. 95-138, 1977.

[13] L. De Lathauwer, B. De, and M. J. Vandewalle, “Computation of the canonical decomposition by means of a simultaneous generalized schur decomposition”, SIAM J. Matrix Anal. Appl., vol. 26, no. 2, pp. 295-327, 2004.

[14] L. De Lathauwer, "A link between the canonical decomposition in multi-linear algebra and simultaneous matrix diagonalization," SIAM J. 
Matrix Anal. Appl., vol. 28, no. 3, pp. 642-666, 2006.

[15] N. D. Sidiropoulos, G. B. Giannakis, and R. Bro, "Blind PARAFAC receivers for DS-CDMA systems,” IEEE Trans. Signal Process., vol. 48, no. 3, pp. 810-823, 2000.

[16] D. L. Donoho. Compressed sensing,.IEEE Transactions on Information Theory, 2006, 52(4): 1289-1306.

[17] E. Candes, J. Romberg, J. Tao. Robust uncertainty principles: Exact signal reconstruction from highly incomplete frequency information. IEEE Transactions on In formation Theory, 2006, 52(2): 489-509.

[18] D. Malioutov, M. Cetin, and A. S. Willsky. "A sparse signal reconstruction perspective for source localization with sensor arrays". IEEE Trans. Signal Process. , 2005, 53, (8):. 3010 - 3022

[19] N. Hu, Z. Ye, D. Xu and S. Cao. "A sparse recovery algorithm for DOA estimation using weighted subspace fitting”, Signal processing, 2012, 92: 2566-2570

[20] R. Bro, N. D. Sidiropoulos, and G. B. Giannakis, “A fast least squares algorithm for separating trilinear mixtures," in Proc. Inter. Workshop ICA and BSS, France, Jan. 1999, pp. 289-294.

[21] P. Stoica and A. Nehorai, "Performance study of conditional and unconditional direction-of-arrival estimation,” IEEE Trans. Signal Process., vol. 38, pp. 1783-1795, Oct. 1990. 